\title{
A conceptional framework of spatial epidemiological research for natural focus diseases, a perspective of disaster science
}

\section{An Zhao}

Jiangxi Normal University

Rui Li (D1061306448@qq.com )

Tsinghua University https://orcid.org/0000-0002-9492-4452

\section{Research}

Keywords: natural focus diseases, perspective of disaster science, universal spatial epidemiological research framework

Posted Date: September 9th, 2020

DOl: https://doi.org/10.21203/rs.3.rs-68506/v1

License: (c) (i) This work is licensed under a Creative Commons Attribution 4.0 International License.

Read Full License 


\section{Abstract}

Objective: To develop a new spatial epidemiological research framework, which would overcome the problems of multicollinearity among the independent variables and the difficulty for outcome to give an explicit explanation for disease transmission mechanism in the current spatial epidemiological methods.

Methods: Comparison of schistosomiasis transmission process with disaster process; Imitation with and import of disaster risk assessment methodology into spatial epidemiological research.

Results: A universal spatial epidemiological research framework was presented for the natural focal disease infection risk prediction. This new approach was expected to be extended to the risk assessment of environmental pollution-incurred public health problems. The GIS-based spatialization methods of the factors concerned in the framework were suggested as well.

Conclusion: The new established spatial epidemiological framework presented has strong universality and shows many advantages in the field of public health.

\section{Introduction}

The 21 st century has witnessed many new health problems: the increasing incidence of chronic diseases which has become a new threat to human health, new and existing infectious diseases and public health emergencies continuing to threaten human health, and constant public rising demands for health etc (Zhou et al, 2015), which pose new challenges to public health work. Natural focus diseases are typical public health problems; some natural ecological environment creates proper conditions for the spread of pathogens among wild animals. People are liable to be infected by pathogens through arthropods or host vertebrates when entering these areas (Luo and Liu, 2007). At present, numerous serious infectious diseases around the world almost all pertain to natural focus diseases.

The epidemic transmission of natural focus diseases has the characteristics of natural focus, regionality and seasonality, the occupational and relatedness with human activities (Wang, 2010), and especially dependence on geographical environment factors. Host, vector and pathogen are the three basic elements of natural focus diseases, which are affected by climate, topography, soil, vegetation and other geographical factors with spatial correlation in causation and mechanism. Existing spatial epidemiological methods usually assumed that the environmental factors associated with the spatial distribution of vectors and pathogens as the independent variables, and the host morbidity as the dependent variables in the mathematical model. These research methods can be divided into two basic categories: classical statistics (Fang et al, 2008; MacMillan, 2012; Wen et al, 2005; Yan et al, 2007) and spatial-temporal model (Steinmann et al, 2007; Wang et al, 2008). There obviously exist two problems in the present methods, the first one is large multi-colinearity among these explanatory variables which have only indirect relationship with the response variable (e.g. morbidity), inevitably leading to information redundancy. The second one is that these environmental factors are generally difficult to give an explicit mechanism explanation for the epidemic transmission process of the disease, which could greatly reduce 
the practicability of the calculation results. Taking the epidemic transmission of schistosomiasis as an example, this paper analyzed the basic elements, their interrelations and their spatialization methods, and established a new research framework to estimate infection risk of natural focus diseases in the field of public health from the perspective of disaster science.

\section{Analysis Of The Basic Elements And Their Interrelationship In Disaster Science}

\subsection{Basic framework of disaster risk assessment}

One of the core issues in disaster science is disaster risk, which is the uncertainty of future loss caused by natural disaster. This system not only reveals the causation of natural disaster risk, but also embodies the natural and social characters of natural disaster risk (Ni and Wang, 2012). The definition of disaster risk and its mathematical expression (formula (1)) given by the Humanitarian Affairs Department of the United Nations in 1992 have been widely accepted by the international community. That was, disaster risk was the severity of adverse events after the interaction of risk sources and vulnerability.

$\mathrm{Dr}=\mathrm{DI} \times \mathrm{Vd}(1)$

where $\mathrm{Dr}$ was the disaster risk, DI was the danger level of the hazard source, $\mathrm{Vd}$ was the vulnerability of the disaster bearing body.

Zhang et al ${ }^{11}$ gave a more comprehensive expression (formula (2)) of the disaster risk, which was introduced into various disaster risk assessment (Zhang et al, 2007; Zhang et al, 2005).

$\mathrm{Dr}=\mathrm{DI} \times \mathrm{Vd} \times \mathrm{Ep} \times \mathrm{Pm}$

where the meanings of DI and Vd were the same as in formula (1); Ep was the exposure degree of the disaster bearing body to hazard source, Pm was the prevention and mitigation countermeasures against the hazard source, these two variables were supplemented to consummate formula (1).

The essential meanings of the two formulas were the same. The factors Ep and Pm in formula (2) can actually be incorporated into the factor Vd in formula (1) in some specific manner (Pelling et al, 2004; Li and $\mathrm{Wu}, 2011)$, but the implication of the formula (2) was much more distinct.

\subsection{Basic elements and their interrelationship in disaster risk assessment}

The natural disaster system can be regarded as an integral whole composed of disaster pregnant environment, disaster causing factors, disaster bearing body (victim) and disaster prevention and mitigation capability. The disaster pregnant environment provides the background conditions for the disaster causing factors, dictating the types, frequency and intensity of the disaster. Disaster bearing body is the weighing instrument of natural disaster risk, its exposure, vulnerability and disaster prevention and mitigation capability directly determine the existence and level of risk. 
Disaster risk assessment is generally measured in the way of expected loss of life or monetary value, which can be calculated by the direct loss and indirect loss respectively. Its characterization can be categorized into three ways: risk chart, risk curve and the formula method (Ma, 2015). The factor of hazard can be described as danger degree of disaster causing factors that may incur casualties, property losses, ecological degradation, social and economic losses. It regularly measures as the strength or probability of hazard. The factor of vulnerability of disaster bearing body refers to the maximum potential damage degree caused by the potential disaster (Liu, 2000), or can be perceived as the sensitivity of the disaster bearing body to damage (Gary, 1997), or the possibility of being damaged by the disaster event (David, 1997; Louis, 1994). The existence of hazard sources does not mean the existence of risk because the risk is relative to the subject - human beings and their socio-economic activities (Su and Gao, 2003). Only when a certain hazard source is likely to affect a certain socio-economic target object and incur obviously adverse consequences, can it really bring about disaster risk. The factor of exposure indicates

whether disaster bearing bodies are within the impact scope of the hazard sources and may suffer damage. If disaster bearing bodies are outside the impact scope of the hazard sources, they are free of disaster risk. The measurement of exposure is to what extent the disaster bearing body is within the influence range of the disaster causing factors. It can be expressed by the ratio of the area, volume or length of the disaster bearing body exposed to the disaster causing factors over its total $(\mathrm{Ma}, 2015)$. The factor of disaster prevention and mitigation capacity is generally expressed as the effectiveness of disaster prevention and mitigation measures, including the validity of engineering and non-engineering disaster prevention measures. Specifically, it refers to the capability to protect and relieve the disaster bearing body from the possible damage through monitoring and prediction system, defense system, emergency rescue system and post disaster recovery and reconstruction for the disaster (Ni, 2013).

\section{Basic Elements In Schistosomiasis Transmission And Their Connotations From The Perspective Of Disaster Science}

\subsection{Basic framework of schistosomiasis infection risk corresponding to disaster risk assessment}

Schistosomiasis is a typical natural focus disease closely associated with geographical factors, bound up with the specific natural and social environment. Determination of the internal relationship between the prevalence of schistosomiasis and its associated factors is the key to control the disease (Yang et al, 2007). Analysis of the basic elements of schistosomiasis transmission is indispensable from the process of schistosome transmission (Zhao, 2016). Eggs of schistosome harbored in the feces of schistosome patients or contractable sick mammals hatch into miracidium at a certain temperature. The miracidium becomes highly activated when there is oncomelania nearby, penetrates into and parasitizes in the body of Oncomelania, therefore, the Oncomelania become infected. Then the miracidium grow into cercariae after a certain period (generally 30-40 days) of development, which can infect the human and livestock. Cercaria spreads in the water, and thus forms epidemic waters. Spatial distribution of epidemic waters and cercariae attachments forms the susceptible area, and the spatial distribution of density and infectivity of the cercariae in the susceptible area thus contributes to schistosome susceptible map 
(susceptibility map). The spatial range and intensity of human and livestock activity in the susceptible area constitute the water contact index map. The GIS (Geographic Information System) overlay spatial analysis of the schistosome susceptible map and the water contact index map will shape up the risk map of schistosomiasis infection.

As a typical natural focus disease, the risk evaluation of schistosomiasis infection has similar structural characteristics with the basic framework of disaster risk assessment. Corresponding to formula (2), there is also following mathematical expression between the risk of schistosomiasis infection and the relevant factors:

Rs $=\mathrm{Ss} \times \mathrm{Wc} \times \mathrm{li} \times \mathrm{Hp}$

where Rs is the risk of schistosomiasis infection, Ss is the susceptibility in susceptible area, Wc is the water contact index, li is the individual acquired immunity, $\mathrm{Hp}$ is the human or animal protection measures against the susceptibility of schistosomiasis infection.

In comparison with the factors in formula (2), the Rs here corresponds to the $\mathrm{Dr}$ (disaster risk); Ss corresponds to the DI (or danger level); Wc corresponds to the Ep (exposure degree of disaster bearing body to hazard source); li corresponds to the Vd (the vulnerability of disaster bearing body); $\mathrm{Hp}$ corresponds to the Pm (prevention and mitigation countermeasures against hazard source).

\subsection{The connotations of the basic elements in schistosomiasis epidemic transmission in parallel with disaster risk assessment}

According to formula (3), the risk of schistosomiasis infection can be defined as the results of different spatial distribution of schistosomiasis susceptibility, different exposure intensity (human and animal activity regularity), differences in individual acquired immunity and protective measures adopted in a certain region.

The susceptibility in susceptible area (Ss) is the potential infectivity or danger degree of pathogen schistosomiasis in nature to human and animal before their contact with epidemic waters in susceptible area, including the water surface, shallow waters, dew on vegetation branches etc that harbor schistosome cercariae and can infect human and animals. The susceptibility can be measured in the forms of density of the cercaria (the pathogen) or positive Oncomelania (the vector) in epidemic waters and its attachments. The cercaria can be substituted by the feces pollution index (FPI) (Wu et al, 1990).

The water contact index $(\mathrm{Wc})$ refers to the contact intensity between the contractable human \& livestock and the source of infection. The Wc can be expressed as following (Qin et al, 2000):

$\mathrm{Wc}=\mathrm{Lc} \times \mathrm{Ca} \times \mathrm{Cf}$ 
where Lc is the length of contact time length; $\mathrm{Ca}$ is the contact body area (or ratio); Cf is the water contact frequency.

Individual acquired immunity (li) refers to the acquired immune capability of individual population or animal to schistosoma cercariae due to different age and body constitution etc, which may lead to slightly different results under the same susceptibility level and contact intensity of the epidemic water.

Human and animal protection measures $(\mathrm{Hp})$ include applying protective cream, wearing protective rubber gloves and boots during activities in susceptible areas. This factor is generally associated with the individual's awareness of the hazards of schistosomiasis infection, prevention awareness, education level, age, warning signs set by the schistosomiasis control department etc.

In comparison of the aforementioned basic elements in disaster science with those in schistosomiasis infection, the disaster causing factor is the pathogenic factor, i.e. schistosome cercariae (or pathogen). The disaster pregnant environment is the relevant environmental factors affecting the spatial distribution of cercariae, including the cercariae attachments, positive oncomelania, in which oncomelania is the unique intermediate host (vector). The disaster bearing body is human and other contractable mammals (hosts). Slightly different from the disaster science, the human and animal in the risk assessment of schistosomiasis infection are not only the victims of schistosomiasis, but are also one of the terminal hosts in the life cycle of schistosomiasis transmission.

\subsection{The spatialization methods of basic elements in schistosomiasis epidemic transmission}

The key of the new research methodology is to spatialize the basic elements involved in schistosomiasis epidemic transmission to generate the factor maps to analyze the spatial distribution of schistosomiasis risk so that the high-risk groups and high-risk areas can be identified.

As the spatial difference of the individual acquired immunity in the above risk assessment expression of schistosomiasis infection is very small, and the human and animal protection measures in local areas are of little disparity, the key factors are the susceptibility in susceptible areas and the contact index with epidemic waters. The substance of susceptibility is the infectivity of schistosome at a specific geographical location, while the nature of water contact index is the degree or proportion of human and animal body exposed to the source of infection. Therefore, the key to study the spatial variation of schistosomiasis infection risk in this framework is the spatialization of susceptibility and water contact index.

The spatialization of susceptibility can be approached by GIS spatial interpolation from cercariae detection point data by means of sentinel mice exposure test. Present GIS has a variety of interpolation methods to address this problem. The accuracy of the interpolation results depends on the sampling scheme of cercariae detection points and interpolation method. However, the schistosome cercariae can drifts and diffuses in flowing water (Xie et al, 2005), wind direction and speed can also affect the spatial distribution and density of cercariae (Zhong et al, 1994). Therefore, cercariae have tendency to 
downstream or to the shore, which results in the spatial dislocation between the pathogen source and the infected area (i.e. susceptible area), possibly making the spatial interpolation results slightly different from the actual spatial distribution of cercariae. To this end, we can use the data of velocity and direction of water flow and wind to modify the spatial interpolation results with the grid neighborhood calculation method of GIS. In addition, cercariae can only survive in specific waters, in the case of spatial interpolation results occurring on land, it can be calibrated by GIS Erase operation over the landcover thematic data.

The spatialization of the epidemic water contact index needs to first analyze the hot spot areas where people and animals may regularly contact epidemic waters, such as the relatively fixed fishing sites of fishermen, or the places where people often forage, wash clothes and vegetables in the marshland schistosomiasis epidemic area. Then, through field and questionnaire survey on the time length, frequency and contacting area of the body with epidemic waters etc, statistical and GIS spatial analysis can be employed to produce the epidemic water contact index map according to formula (4).

\section{A New Conceptional Framework Of Spatial Epidemiological Research}

It can be seen from the analysis, the risk of schistosomiasis infection can actually be studied in a new framework (formula (3)) of spatial epidemiological research that imitates the basic framework of disaster risk assessment.

In addition, the new framework of disease infection risk is not only suitable for the spatial epidemiological research of natural focus diseases, but also can be used for the risk assessment of the public health problems caused by environmental pollution. Corresponding to the previous formulas (2) and (3), a similar risk assessment framework for environmental pollution hazards can be established as follows:

$R e=H p \times E p \times I p \times P p$

where $\mathrm{Re}$ is the morbidity risk subjecting to the type of environmental pollution, $\mathrm{Hp}$ is the intensity of hazardous pollutants (concentration, toxicity etc), Ep is the degree of exposure to pollutants (frequency, time etc), Ip is the individual physical fitness (physically metabolic capacity to pollutants etc), Pp is the protective measures against pollutants.

\section{Discussion}

Taking schistosomiasis as an example, this paper explored a new spatial epidemiological framework for study on the risk of natural focus diseases imported from the framework of disaster risk assessment. Although the new framework is of general applicability to most natural focus diseases, the transmission process and route of a specific disease are not completely the same among the wide variety of natural focus diseases. The basic elements of a disease, their interrelationship and spatialization methods are 
also different albeit in the similar way as in the disaster risk assessment framework. The general steps to use the new spatial epidemiological methods to study natural focus diseases is as follows. Firstly, GIS layers of relevant vector-harboring environmental factors for pathogen breeding need to be established respectively according to the targeted specific disease. Secondly, these factor GIS layers can be used to produce the pathogen hazard GIS layer by spatial analysis (such as buffer analysis, spatial interpolation, grid neighborhood calculation etc). Thirdly, the possibility and degree (intensity) of host exposure to hazardous pathogen can be determined according to the biological relationship between pathogen and host. This exposure index may be expressed in different ways for different natural focus diseases, it needs to determine the exposure hot spots, supplemented with sampling survey data, and use of GIS (e.g. overlay analysis, geographic network analysis, cellular automata etc). Finally, the product of the GIS layer "pathogen hazard" and "exposure index" is the disease infection risk map. Respecting with the extension of this new frame to the risk assessment on the public health problems caused by environmental pollution, the spatialization methods of the basic factors are different depending on the type of pollutants.

Compared with other spatial epidemiological methods in the field of public health, the advantages of this new research framework are as follows. First, the multicollinearity of the independent variables is generally weak because in terms of diseases transmission process, each independent variable in the formula is a linkage in the disaster process. Secondly, each independent variable has a clearly explicit connotation in contrast with using the implicit environmental factors as predicting factors in the present mathematical models. Thirdly, the spatial resolution of the calculation results is high (at grid level), which can predict the epidemic risk at a specific geographic location and pinpoint to the possible disease outbreak hot spot area. Fourthly, if we have the data of the number of patients or prevalence rate at a certain amount of specific sampling points, we can establish regressive relation between the epidemic risk and the number of patients or the prevalence rate for the whole area. Likewise, if we have the data of the number of patients or the average prevalence rate of the whole area, we can compute the corresponding values at each grid location through areal interpolation method (Michael, 2007; Dong et al, 2016) of GIS. In short, the methodological framework proposed in this paper is expected to become a new spatial epidemiological research approach in the future public health research.

\section{Declarations}

\section{ACKNOWLEDGMENTS}

This work was supported by funding from the National Natural Science Foundation of China [81260449].

\section{CONFLICTS OF INTEREST}

None of the authors has any conflict of interest.

\section{AUTHOR CONTRIBUTIONS}


An Zhao: Conceptualization, Writing - original draft

Rui Li: Conceptualization

\section{References}

David A, 1997. The study of natural disasters (1977-1997): Some reflections on a changing field of knowledge. Disasters, 21(4): 284-304.

Dong N, Yang XH, Cai HY, 2016. Research progress in spatialization of population data. Journal of Earth Information Science, 18: 1295-1304. (in Chinese)

Fang LQ, de Vlas SJ, Liang S, 2008. Environmental factors contributing to the spread of H5N1 avian influenza in mainland China. PLoS One,2008, 3: e2268区

Gary S, 1997. An assessment of disaster risk and its management in Hailand. Disasters, 21: 77-88.

Li N, Wu JD, 2011. Introduction to natural disaster emergency. Beijing: Peking University Press, 105-128. (in Chinese)

Liu XL, 2000. Study on risk assessment of regional landslide. Journal of natural disasters, 9: $54-61$. (in Chinese)

Louis S, 1994. Urban developments and megacities: Vulnerability to natural disasters. Disaster Management, 6(3):160-169.

Luo CW, Liu QY, 2007. Analysis of epidemic factors of natural focus diseases and countermeasures. Chinese Journal of vector biology and control, 18: 293-297. (in Chinese)

Ma BC, 2015. Definition and characterization of natural disaster risk. Disaster science, 30: 016-20. (in Chinese)

MacMillan K, Monaghan AJ, Apangu T, 2012. Climate predictors of the spatial distribution of human plague cases in the West Nile region of Uganda. American Journal of Tropical Medicine and Hygiene, 86区 514-523.

Michael R. Geographic Information Systems and Spatial Data Processing in Demography: a Review. Population Research And Policy Review $₫ 2007$ 26:601-618.

$\mathrm{Ni} \mathrm{CJ}$, Wang J. On the re-definition of natural disaster risk. Disaster science, 2012, 27: 1-5. (in Chinese)

Ni CJ. Approach to natural disaster risk assessment. Disaster science, 2013,28: 1-5. (in Chinese)

Pelling M, Maskrey A, Ruiz P, et al. United Nations Development Programme. A global report reducing disaster risk: A challenge for development. NewYork: UNDP, 2004囚1-146. 
Qin XX, Xiao X, Wang $\mathrm{H}$ et al: A prospective study on re-infection of Schistosoma japonica in a River beach type epidemic area. Chinese Journal of schistosomiasis control, 2000,12: 273-276. (in Chinese)

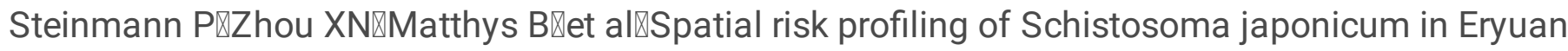

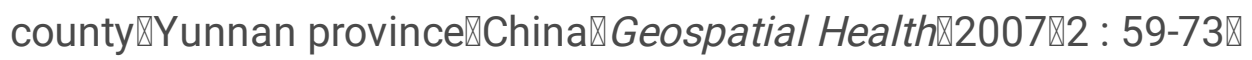

Su GW, Gao QH. Issues on characteristics of subject behavior and the time scale for natural disaster risk. Journal of natural disasters, 2003, 12: 9-16. (in Chinese)

Wang CJ, Zhang JH, Gu HT, et al. Epidemic characteristics of natural focus diseases and key points for handling outbreaks. Practical preventive medicine, 2010, 17: 202-205. (in Chinese)

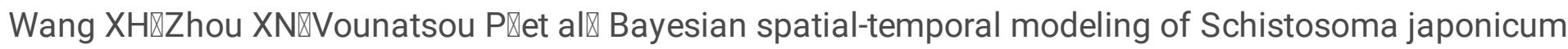

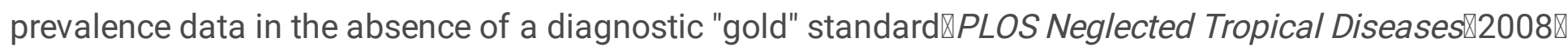
2: e250区

Wen L, Xu DZ, Wang SQ, et al. Study on the correlation between satellite remote sensing vegetation index and the distribution of malaria endemic areas in Hainan Province. Chinese Journal of epidemiology, 2005, 26: 263-267. (in Chinese)

Wu ZW, Zhuo SJ, bu KM, et al: Calculation and application value of actual pollution index of various schistosomiasis infectious sources in the Dongting Lake area. Chinese Journal of schistosomiasis control, 1990, 2: 11-14. (in Chinese)

Xie CY, Gao Y, Qiu L, et al: Observation of sentinel mouse method to determine the susceptibility zone of schistosomiasis, Chinese Journal of Parasitology and Parasitology, 2005, 23: 315-316. (in Chinese)

Yan LXFang LQ囚Huang HG囚et al『Landscape elements and Hantaan virus-related hemorrhagic fever with

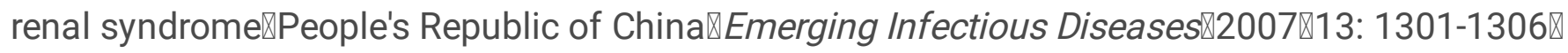

Yang, K., Wang, X.H., Wu, X.H., et al. (2007). Application of spatial epidemiological techniques in the prevention and control of schistosomiasis. China public health, 23: 1017-1019. (in Chinese)

Zhang, H., Zhang, J.Q., Han J.S. (2005). Risk assessment and regionalization of flood disaster based on GIS technology - Taking the middle and lower reaches of the Liaohe River as an example. Journal of natural disasters, 14: 141-146. (in Chinese)

Zhang, J.Q., Li, N. (2007). Quantitative methods and application of risk assessment and management of major meteorological disasters. Beijing: Beijing Normal University Press, 20-36. (in Chinese)

Zhang, J.Q., Liu, X.P., Tong, Z.J. (2007). Grassland fire risks assessment and zonation. Geographic research, $26: 755-762$. (in Chinese) 
Zhao, A. (2016). Application of geo information technology in the study of medical geography of schistosomiasis. Beijing: Higher Education Press, 8-15. (in Chinese)

Zhong, J.H., Zhang, S.J., Liu, Z.D. (1994). The relationship between acute schistosomiasis and susceptible areas in Poyang Lake area, Chinese Journal of Parasitology and parasitic diseases, 12: 6667. (in Chinese)

Zhou, H.X., Tang, X.Y., Qiu, X.Q. (2015). Current situation and prospect of spatial epidemiology, theory and method. Foreign medical geography, 36: 79-92. (in Chinese) 